Research Paper

\title{
Association of HLA-DRB1, HLA-DQB1 Polymorphisms with HPV 16 E6 Variants among Young Cervical Cancer Patients in China
}

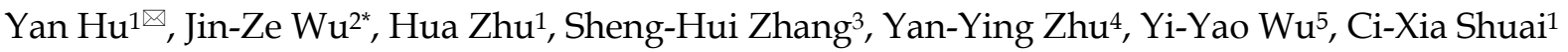 \\ 1. Department of Gynecology, 1st Affiliated Hospital, Wenzhou Medical University, Wenzhou, Zhejiang, P.R.China; \\ 2. Department of Anesthesiology, 1 ${ }^{\text {st }}$ Affiliated Hospital, Wenzhou Medical University, Wenzhou, Zhejiang, P.R.China; \\ 3. Department of Hematology, 1st Affiliated Hospital, Wenzhou Medical University, Wenzhou, Zhejiang, P.R.China; \\ 4. Laboratory Diagnosis Center, $1^{\text {st }}$ Affiliated Hospital, Wenzhou Medical University, Wenzhou, Zhejiang, P.R.China; \\ 5. College of Medicine, Jiaxing University, Jiaxing, Zhejiang, P.R.China. \\ *Equal contributors and co-first authors.
}

$\triangle$ Corresponding author: Yan Hu, Department of Gynecology, 1st Affiliated Hospital, Wenzhou Medical University, Shangcai Cun, Nanbaixiang, Ouhai District, Wenzhou City, Zhejiang Province, 325000, P.R.China. Tel:008657755579165; Fax:008657755579555; E-mail: drhuyan@wzhospital.cn

(c) Ivyspring International Publisher. This is an open access article distributed under the terms of the Creative Commons Attribution (CC BY-NC) license (https:// creativecommons.org/licenses/by-nc/4.0/). See http://ivyspring.com/terms for full terms and conditions.

Received: 2017.02.25; Accepted: 2017.05.14; Published: 2017.07.22

\begin{abstract}
Potential correlation of human papillomavirus (HPV) 16 E6 variants and human leukocyte antigen (HLA) class II polymorphisms has been suggested in patients with cervical cancer, so far little information is available about the possible interaction between E6 variants and HLA class II variability during the obviously accelerated progression to cervical cancer in young women. In this study, we aimed to explore the association between the HPV16 E6 variants and HLA-DRB1, DQB1 alleles in a Chinese young cervical cancer population. The HLA-DRB1, HLA-DQB1 polymophisms were genotyped by low-resolution polymerase chain reaction (PCR) with sequence-specific primer. HPV16 E6 DNA was tested by Sanger fluorescent dye dideoxy-termination technique. The difference of DRB1, DQB1 polymorphisms between young cervical cancer patients $(\leq 35 y s, n=61)$ and older ones $(>35 y s, n=85)$ and the association with E6 variants were analyzed. Results showed that the distribution pattern of HLA-DRBI, DQBI alleles was different between young cervical cancer patients and older ones. The allele frequency of $\mathrm{DQB1} * 0501$ in young patients was significantly lower than older ones $(6.6 \%$ vs. $23.5 \%, p<0.05)$. The HPV16 E6 A4 lineage was the exclusive type observed in young patients, and its prevalence was significantly higher than that of older cases $(82.86 \%$ vs.41.94\%, $p<0.05)$. DRB1*03 was not found in young patients positive for the HPV16 E6 A4 lineage, whereas it was observed in $19.2 \%$ older patients with A4 positive $(\mathrm{Pc}<0.05)$. In conclusion, specific association between certain HPV16 E6 variant and genetic polymorphisms of HLA may play a role during the progression of early onset cervical cancer in young patients. Certain HLA-DRBI and HLA-DQB1 alleles may be related to the A4 lineage among young cervical cancer patients, which was the unique HPV16 E6 variant found in Chinese young patients. Our finding may provide an insight into the pathogenic factors that associated with cervical cancer in young women.
\end{abstract}

Key words: human papillomavirus; human leukocyte antigen; polymorphism; variant; age

\section{Introduction}

According to WHO statistics, invasive cervical cancer (ICC) still ranks as the fourth most prevalent cancer in women and more than $80 \%$ cases arise in developing countries [1,2]. In recent years, the worldwide incidence and mortality of ICC have generally decreased, owing to the successful implement of cervical cancer screening program and great achievements in early diagnosis and treatment of premalignant conditions. However, the incidence of ICC in young women has been reported to be 
increasing steadily both in China and in other countries [3-7]. In most reports, young women seemed to have a more aggressive form of the disease and a poorer prognosis than older women [3-6]. Therefore, studies aimed to investigate the possible pathogenic factors responsible for young patients with ICC are of great concern for their early prevention.

Genital infection of human papillomaviruses (HPVs), particularly the persistent infection status of high-risk HPVs is recognized as a causal and necessary factor in the pathogenesis of ICC. It is well known that high-risk HPV infection is very popular among women at reproductive age. Human papillomavirus early proteins E6 and E7 subvert normal cell control mechanisms through interactions with two oncosuppressor proteins, p53 and $\mathrm{Rb}$, respectively [8]. Human papillomavirus type 16 infection was found to account for about $50 \%$ of ICC worldwide $[2,9,10]$. However, majority of high-risk HPV infections are transient and disappear spontaneously, only a small percentage of HPV infections persist and progress to precancerous diseases or ICC, indicating that additional factors are needed during the pathogenic process. Both viral and host genetic factors of infected individuals were considered to be important factors in determining progression to neoplasia of high-risk HPV infection.

Recently, different HPV16 E6 variants were observed to be related to an increased risk for cervical ICC in a certain population [11-13]. Distinct geographic diversity was observed in the distribution of HPV16 E6 variants. Currently, the variants were classified into four lineages [14]: A, which includes A1-A3 (previously called European, EUR) and A4 (previously named Asian, As); B (previously named African 1, AF-1); C (previously named African 2, AF-2); D, which includes Asian-American (AA) and North-American (NA) lineages named previously. Based on nucleotide polymorphism at nt350, the A1-A3 lineage were further classified into 350T and 350G.The A4 lineage was seldom reported in the west, but in some Asian countries such as Japanese, Korean and the southern area of China, it has becoming the most common variant found in cervical cancer cases [15-18]. We investigated the distribution of HPV16 E6 variants in cervical squamous cell carcinoma (SCC) patients from a coastal city located in the southern part of China and found that the A4 lineage was the most common variant type [19]. In addition, we also found that the distribution of HPV16 E6 variants was significantly different between younger cervical SCCs ( $\leq 35 y s)$ and older patients (>35ys). In young patients group, we only detected the A4 lineage. However, other linages of HPV 16 E6 variants were found in the older cervical SCCs in addition to the A4 lineage. The unmatched distribution of the A4 lineage observed in young SCC patients indicated the possible oncogenicity of this variant type might be more aggressive in young cervical SCCs of this study population.

On the other hand, the host genetic diversity may also play an important part in the natural process of HPV infections. The distinct characteristics of HPV16 E6 variants distribution observed in cervical diseases among ethnically different populations suggest a possible relationship between E6 variants and human leukocyte antigens (HLA), which are population related. As we know, HLA plays a key role during the clearance of virally infected cells by presenting xenogenic antigens to the cytotoxic T cells. The highly polymorphic character of HLA genes may display different ability in the clearance of HPV-infected cells, and hence the progression to cervical cancer. HLA class II provides the main genetic susceptibility loci to cervical cancer. The strongest effects are from the HLA II DQB1 and DRB1 loci $[20,21]$. Several researches have analyzed the possible correlation of HPV16 E6 variants with HLA class II polymorphisms in patients with ICC from different populations and found certain HLA alleles showed to be correlated to a changed risk for ICC among different ethnic population [22-24]. Although our previous study has implied that the uniform presence and distinct raised E6 A4 lineage in young age cervical SCCs may be closely related to the early occurred cervical SCC, so far little information is available about the HLA class II polymorphism in relation with the HPV16 E6 variants in young women aged 35 or younger with cervical SCC. In the present study, we examined the difference of DRB1, DQB1 polymorphisms between young cervical SCCs and older ones and the association with E6 variants, with the aim of discovering the possible interaction between E6 variants and HLA class II variability during the obviously accelerated progression to cervical cancer in young women.

\section{Materials and Methods}

\section{Study Population}

The specimens of cervical SCCs in this study were collected at the Gynecology ward of 1st Affiliated Hospital of Wenzhou Medical University between June 2009 and June 2015. Sixty-five women aged $\leq 35$ while 713 patients aged $\geq 36$ at the time of diagnosis. All members of this study had access to identifying information during data collection. Each patient enrolled in this study needed to be a native resident in Wenzhou city, which is a coastal city 
located in the south of China. Sixty-one women aged $\leq 35$ were enrolled in group I, 85 patients aged $\geq 36$ (selected by an 8-patient interval sampling design) in group II. The cut-off limit of 35 years for young cervical cancer has been applied by many researchers $[3,6]$. The mean ages of the two groups were $32.4 \pm 2.9$ years and $51.1 \pm 6.7$ years respectively. At the same time, we invited 130 age-matched healthy volunteers who came for routing cervical disease screening to take part in our study as healthy controls to provide their cervical cellular specimen and blood samples. The ethnicity, marital status, and smoking habits of controls were similar to the patients. The average age of the control group was $39.8 \pm 10.3$ years.

\section{Research Process and Sample Collection}

The research plan was permitted by the research ethics committee of the 1st Affiliated Hospital of Wenzhou Medical University (20040912). All patients and controls gave written informed consent. The medical, gynecological, sexual and childbearing history were provided by all patients and controls. Physical and gynecological examinations were performed afterwards. For all cervical SCC patients, peripheral serum samples and cervical cytology samples were collected before surgical treatment. The cervical cytology samples were sent for HPV genotyping. Their cervical tissue samples were collected during the surgery and were placed immediately in liquid nitrogen until required for analysis. Of the healthy controls, peripheral serum samples and cervical cytology samples were collected. The cervical cytology samples were sent for Liquid-based cytological test and HPV genotyping. Blood samples drawn from all cervical SCCs and healthy controls were analyzed for DRB1 and DQB1 loci.

\section{HPV Genotyping}

The cervical cytology samples of all cervical SCCs and healthy controls were examined for HPV genotypes as described earlier [19].

\section{HPV 16 E6 Variants Analysis}

The cervical DNA samples were extracted in HPV16 positive cervical SCC patients and sequenced as described earlier [19]. The BLAST 2.0 software server (http://www.ncbi.nih.gov/blast) was used to analyze DNA sequence. The variants were classified into four lineages as A (A1-3, A4), B, C and D. Based on nucleotide polymorphism at nt350, the A1-3 lineage were further classified into 350T and 350G.

\section{Human Leukocyte Antigen Typing}

Genomic DNA of patients and healthy controls were isolated from peripheral venous blood samples by using the DNeasy Blood \& Tissue Kit (QIAGEN, Germany). Genotyping of HLA-DRB1 and HLA-DQB1 as low-resolution allele distribution was determined by PCR sequence specific primers (SSP) method. DRB1and DQB1 PCR-SSP Morgan ${ }^{\mathrm{TM}}$ Kits were supplied by Texas BioGene Inc. (Texas, USA). Amplification reactions were done according to manufacturer's protocols. Results were photographed with a gel-imaging system and analyzed by Morgan ${ }^{\mathrm{TM}}$ SSPal HLA Typing Analysis Software.

\section{Statistical Analyses}

Statistical analyses were conducted using Statistical Package for the Social Sciences (SPSS) 22.0 software. Allele frequencies of DRB1and DQB1 were calculated by direct counting. Chi-squared $\left(x^{2}\right)$ comparisons were performed to identify any differences on categorical variables. Odds ratios (ORs), 95\% confidence intervals (95\% CIs) and $p$ values were calculated. According to Bonferroni's correction, corrected $p$ value (Pc) was calculated for multiple comparisons, which multiplied the $p$ value by the number of alleles tested for each locus. All $p$ values were assumed significant as $p<0.05$.

\section{Results}

\section{Characteristics of the Cervical SCC Patients}

Demographic characteristics between two cervical SCC groups were compared (Table 1). No significant difference was found except for the number of pregnancies.

Table 1. Comparison of demographic characteristics between young cervical SCC patients and older ones.

\begin{tabular}{lll}
\hline Characteristics & Group I $(\mathbf{n}=\mathbf{6 1})$ & Group II $(\mathbf{n}=\mathbf{8 5})$ \\
\hline Ethnicity & $100 \%$ Han population & $100 \%$ Han population \\
Mean age & $32.4 \pm 2.9$ years & $51.1 \pm 6.7$ years \\
Mean age at first & $20.6 \pm 1.7$ years & $21.1 \pm 2.2$ years \\
intercourse & & \\
Sexual partner numbers & & \\
$\mathbf{1}$ & $86.7 \%$ & $85.1 \%$ \\
$\mathbf{1}$ & $13.3 \%$ & $14.9 \%$ \\
Number of pregnancies & $1 \sim 3$ & $1 \sim 8$ \\
Smoking habits & & \\
Yes & $2.3 \%$ & $3.1 \%$ \\
No & $97.7 \%$ & $96.9 \%$ \\
\hline
\end{tabular}

Details of HPV genotype in cervical cytology samples of 146 cervical SCCs and 130 healthy controls were summed up (Table 2). The infection rate of HPV was $96.6 \%(141 / 146)$ and $11.5 \%(15 / 130)$ in cervical SCCs and healthy controls respectively. The most common genotype found in this population was HPV 16. $45.21 \%(66 / 146)$ of cervical SCC patients and $4.6 \%$ (6/130) of controls were HPV 16 positive. The percentage of HPV16-positive patients in group I $(57.4 \%, 35 / 61)$ was significantly higher than that of 
group II $(36.5 \%, 31 / 85)\left(x^{2}=6.267, p<0.05, \mathrm{OR}=2.345\right.$, 95\% CI: 1.197-4.595).

Table 2. Comparison of HPV Genotyping between Cervical SCCs and Healthy Controls.

\begin{tabular}{|c|c|c|c|c|}
\hline \multirow[t]{2}{*}{ HPV Genotype } & \multicolumn{2}{|c|}{ Cervical SCCs } & \multicolumn{2}{|c|}{ Healthy Controls } \\
\hline & $\mathbf{N}$ & $\%$ & $\mathbf{N}$ & $\%$ \\
\hline \multicolumn{5}{|l|}{ Single Infection: } \\
\hline \multicolumn{5}{|l|}{ high-risk type } \\
\hline 16 & 57 & 40.4 & 6 & 40.0 \\
\hline 18 & 9 & 6.4 & 1 & 6.7 \\
\hline 52 & 13 & 9.2 & 3 & 20.0 \\
\hline 58 & 12 & 8.5 & 1 & 6.7 \\
\hline 31 & 10 & 7.1 & 0 & 0.0 \\
\hline 33 & 8 & 5.7 & 1 & 6.7 \\
\hline 45 & 4 & 2.8 & 0 & 0.0 \\
\hline 51 & 3 & 2.1 & 0 & 0.0 \\
\hline 56 & 1 & 0.7 & 0 & 0.0 \\
\hline 35 & 2 & 1.4 & 0 & 0.0 \\
\hline 59 & 1 & 0.7 & 0 & 0.0 \\
\hline 39 & 3 & 2.1 & 1 & 6.7 \\
\hline 68 & 2 & 1.4 & 0 & 0.0 \\
\hline \multicolumn{5}{|l|}{ probable high-risk type } \\
\hline 53 & 0 & 0.0 & 0 & 0.0 \\
\hline 66 & 1 & 0.7 & 0 & 0.0 \\
\hline \multicolumn{5}{|l|}{ low-risk type } \\
\hline $6 / 11 / 42 / 43 / 44 / 81$ & 3 & 2.1 & 1 & 6.7 \\
\hline \multicolumn{5}{|l|}{ Multiple Infection with type 16} \\
\hline $16+52$ & 3 & 2.1 & 0 & 0.0 \\
\hline $16+18$ & 2 & 1.4 & 0 & 0.0 \\
\hline $16+33$ & 2 & 1.4 & 0 & 0.0 \\
\hline $16+58$ & 1 & 0.7 & 0 & 0.0 \\
\hline $16+31$ & 1 & 0.7 & 0 & 0.0 \\
\hline Multiple Infection without type 16 & 3 & 2.1 & 1 & 6.7 \\
\hline
\end{tabular}

\section{Characteristics of HPV16 E6 variants}

66 cervical SCCs and 6 healthy controls with HPV16 infection were further determined for E6 sequence variation (Table 3 ).

The A4 lineage was the most predominant variant found in this study. $63.6 \%(42 / 66)$ cervical SCCs and $16.7 \%(1 / 6)$ healthy controls contained this variant. The other non-synonymous variations detected were the B and A1-3 lineages. The E6 variants distribution was further analyzed in cervical SCCs. In group I, the A4 lineage was the unique variant detected. In addition to the A4 lineage, the B and A1-3 lineages were also observed in group II. Although the A4 lineage was the most common variant observed in both groups, the distribution was obviously different between the two groups. The occurrence rate was $82.9 \%(29 / 35)$ in group I, whilst $41.9 \%(13 / 31)$ in group II $\left(x^{2}=11.897, p<0.05\right)$.

\section{Analysis of HLA-DRB 1 and HLA-DQB 1 Polymorphisms}

Data of the HLA-DRB1 and HLA-DQB1 alleles from 146 cervical SCCs and 130 healthy controls were summarized (Table 4). Hardy-Weinberg equilibrium was found in the allele frequencies of the 2 loci among all healthy controls. The most frequent allele for the HLA-DRB1 loci found in cervical SCCs was DRB1*09 (15.1\%, 44/292), whilst DRB1*15 (13.5\%, 35/260) in healthy controls. The allele frequencies among cervical SCCs were obviously higher than that among healthy controls for DRB1*11 and DQB1*0601 alleles, with a Pc value of 0.013 in the former and 0.016 in the latter. However, the allele frequencies for DRB1*13 and DQB ${ }^{*} 02$ were significantly decreased in cervical SCCs as compared with healthy controls, with a PC value of 0.000 in the former and 0.04 in the latter. Comparison of the other DRB1 and DQB1 allele frequencies between cervical SCCs and healthy controls revealed no significant statistical difference $\left(p / \mathrm{Pc}_{\mathrm{C}}>0.05\right)$.

Meanwhile, differences of DRB1 and DQB1 polymorphisms distribution between HPV16-positive cervical SCCs and healthy controls were compared simultaneously (Table 4). Among HPV16-positive cervical SCCs, the most frequent alleles for DRB1 and DQB1 loci observed were DRB1*08 $(17.4 \%, 23 / 132)$ and DQB1*0601 $(22.7 \%, 30 / 132)$. Negative associations were found for DRB1*13 and DQB1*02 alleles with respect to HPV16-positive cervical SCCs, with a Pc value of 0.026 in the former and 0.04 in the latter. DQB1*0601 allele was observed to be positively related to HPV16 infection, which occurred significantly more often in HPV16-positive cervical SCCs $(\mathrm{Pc}=0.000)$. In addition, a positive relationship of DRB1*11 with HPV16-positive cervical SCCs was suggestive $(p=0.017)$, but no significant difference was kept after Bonferroni's correction $\left(\mathrm{P}_{\mathrm{c}}=0.221\right)$. No significance was observed for the other DRB1/ DQB1 alleles in HPV16-positive cervical SCCs ( $\left.p / \mathrm{Pc}_{\mathrm{C}}>0.05\right)$.

Table 3. Comparison of E6 Variants between Cervical SCCs and Healthy controls.

\begin{tabular}{|c|c|c|c|c|c|c|c|c|c|c|c|c|}
\hline \multicolumn{7}{|c|}{ Nucleotide position } & \multirow{2}{*}{$\begin{array}{l}\text { Predicted amino acid } \\
\text { changes }\end{array}$} & \multirow[t]{2}{*}{ Variants } & \multicolumn{2}{|c|}{ Cervical SCCs } & \multirow{2}{*}{$\begin{array}{l}\text { Healthy } \\
\text { controls } \\
\mathbf{N}(\%)\end{array}$} & \multirow{2}{*}{$\begin{array}{l}\text { Total } \\
\text { N (\%) }\end{array}$} \\
\hline 168 & 178 & 241 & 335 & 350 & 393 & 442 & & & $\begin{array}{l}\text { Group I } \\
\text { N (\%) }\end{array}$ & $\begin{array}{l}\text { Group II } \\
\text { N (\%) }\end{array}$ & & \\
\hline $\mathrm{C}$ & $\mathrm{T}$ & $\mathrm{T}$ & $\mathrm{C}$ & $\mathrm{T}$ & G & A & - & A1-3(350T) & $6(17.1)$ & $5(16.1)$ & $3(50.0)$ & 14(19.4) \\
\hline- & - & G & - & - & - & - & - & $\mathrm{A} 1-3(350 \mathrm{~T})$ & 0 & $2(6.5)$ & $1(16.7)$ & $3(4.2)$ \\
\hline- & G & - & - & - & - & - & $\mathrm{D} 25 \mathrm{E}$ & $\mathrm{A} 4$ & $23(65.7)$ & $12(38.7)$ & $1(16.7)$ & $36(50.0)$ \\
\hline- & G & - & - & - & $\mathrm{T}$ & - & D25E/E97D & $\mathrm{A} 4$ & $6(17.1)$ & $1(3.2)$ & 0 & $7(9.7)$ \\
\hline - & - & - & - & G & - & - & L83V & $\mathrm{A} 1-3(350 \mathrm{G})$ & 0 & $5(16.1)$ & $1(16.7)$ & $6(8.3)$ \\
\hline- & G & - & - & G & - & - & $\mathrm{T} 22 \mathrm{~S} / \mathrm{L} 83 \mathrm{~V}$ & $\mathrm{~A} 1-3(350 \mathrm{G})$ & 0 & $1(3.2)$ & 0 & $1(1.4)$ \\
\hline - & - & - & $\mathbf{T}$ & - & - & C & H78Y/E113D & B & 0 & $5(16.2)$ & 0 & $5(6.9)$ \\
\hline
\end{tabular}


Table 4. HLA-DRB1 and HLA-DQB1 Allele Frequencies among Cervical SCCs and Healthy Controls.

\begin{tabular}{|c|c|c|c|c|c|c|c|c|c|c|c|}
\hline \multirow[t]{2}{*}{ Alleles } & \multirow{2}{*}{$\begin{array}{l}\text { Healthy } \\
\text { Controls } \\
2 n=260 \\
(\%)\end{array}$} & \multirow{2}{*}{$\begin{array}{l}\text { Cervical } \\
\text { SCCs } \\
2 \mathrm{n}=292 \\
(\%)\end{array}$} & \multirow{2}{*}{$\begin{array}{l}\text { HPV16+ } \\
\text { SCCs } \\
2 n=132 \\
(\%)\end{array}$} & \multirow[t]{2}{*}{$\begin{array}{l}\text { Group I } \\
2 \mathrm{n}=122 \\
(\%)\end{array}$} & \multirow[t]{2}{*}{$\begin{array}{l}\text { Group II } \\
2 n=170 \\
(\%)\end{array}$} & \multicolumn{2}{|c|}{$\begin{array}{l}\text { Cervical SCCs } \\
\text { vs } \\
\text { Healthy Controls }\end{array}$} & \multicolumn{2}{|c|}{$\begin{array}{l}\text { HPV16+ SCCs } \\
\text { vs } \\
\text { Healthy Controls }\end{array}$} & \multicolumn{2}{|c|}{ Group I vs Group II } \\
\hline & & & & & & $p$ & OR $(95 \% C I)$ & $p$ & OR $(95 \% C I)$ & $p$ & OR $(95 \% \mathrm{CI})$ \\
\hline \multicolumn{12}{|l|}{ DRB1 } \\
\hline${ }^{*} 01$ & $8(3.1)$ & $2(0.7)$ & $1(0.8)$ & $0(0.0)$ & $2(1.2)$ & 0.074 & $4.603(0.969 \sim 21.876)$ & 0.275 & $4.159(0.515 \sim 33.608)$ & - & - \\
\hline *03 & $23(8.8)$ & $25(8.6)$ & $9(6.8)$ & $11(9.0)$ & $14(8.2)$ & 0.906 & $1.036(0.573 \sim 1.875)$ & 0.488 & $1.326(0.595 \sim 2.954)$ & 0.814 & $1.104(0.483 \sim 2.523)$ \\
\hline *04 & $16(6.2)$ & $24(8.2)$ & $10(7.6)$ & $15(12.3)$ & $9(5.3)$ & 0.350 & $0.732(0.380 \sim 1.411)$ & 0.593 & $0.800(0.353 \sim 1.815)$ & $\underline{0.032^{\mathrm{a}}}$ & $2.508(1.059 \sim 5.937)$ \\
\hline *07 & $21(8.1)$ & $17(5.8)$ & $8(6.1)$ & $3(2.5)$ & $14(8.2)$ & 0.296 & $1.421(0.733 \sim 2.757)$ & 0.471 & $1.362(0.586 \sim 3.163)$ & $\overline{0.038^{b}}$ & $0.281(0.079 \sim 1.000)$ \\
\hline$* 08$ & $29(11.2)$ & $38(13.0)$ & $23(17.4)$ & $14(11.5)$ & $24(14.1)$ & 0.504 & $0.839(0.501 \sim 1.405)$ & 0.084 & $0.595(0.329 \sim 1.076)$ & $\overline{0.508}$ & $0.789(0.390 \sim 1.595)$ \\
\hline *09 & $26(10.0)$ & $44(15.1)$ & $19(14.4)$ & $25(20.5)$ & 19(11.2) & 0.074 & $0.626(0.374 \sim 1.050)$ & 0.197 & $0.661(0.351 \sim 1.244)$ & $\underline{0.028}{ }^{c}$ & $2.048(1.071 \sim 3.919)$ \\
\hline${ }^{*} 10$ & $8(3.1)$ & $4(1.4)$ & $2(1.5)$ & $1(0.8)$ & $3(1.8)$ & 0.170 & $2.286(0.680 \sim 7.681)$ & 0.354 & $2.063(0.432 \sim 9.858)$ & $\overline{0.079}$ & $3.220(0.816 \sim 12.706)$ \\
\hline$* 11$ & $10(3.8)$ & $34(10.7)$ & $13(9.8)$ & $13(11.5)$ & $21(12.4)$ & $\underline{0.001^{\mathrm{d}}}$ & $0.304(0.147 \sim 0.627)$ & $\underline{0.017 \mathrm{e}}$ & $0.366(0.156 \sim 0.859)$ & 0.656 & $0.846(0.406 \sim 1.764)$ \\
\hline${ }^{*} 12$ & $29(11.2)$ & $42(14.4)$ & $18(13.6)$ & $18(14.8)$ & $24(14.1)$ & 0.258 & $0.747(0.451 \sim 1.239)$ & 0.475 & $0.795(0.424 \sim 1.4920$ & 0.879 & $1.053(0.544 \sim 2.039)$ \\
\hline$* 13$ & $18(6.9)$ & $2(0.7)$ & $0(0.0)$ & $2(1.6)$ & $0(0.0)$ & $\underline{0.000}^{\mathrm{f}}$ & $10.785(2.478 \sim 46.945)$ & $\underline{0.002 \mathrm{~g}}$ & $0.931(0.900 \sim 0.962)$ & - & - \\
\hline${ }^{*} 14$ & $21(8.1)$ & $16(5.5)$ & $7(5.3)$ & $3(2.5)$ & $13(7.6)$ & $\overline{0.268}$ & $1.461(0.745 \sim 2.865)$ & $\overline{0.314}$ & $1.569(0.649 \sim 3.792)$ & 0.055 & $0.304(0.085 \sim 1.093)$ \\
\hline$* 15$ & $35(13.5)$ & $31(10.6)$ & $16(12.1)$ & $14(11.5)$ & $17(10.0)$ & 0.304 & $1.310(0.782 \sim 2.192)$ & 0.709 & $1.128(0.599 \sim 2.123)$ & 0.686 & $1.167(0.552 \sim 2.467)$ \\
\hline *16 & $16(6.2)$ & $13(4.45)$ & $6(4.5)$ & $3(2.5)$ & $10(5.9)$ & 0.371 & $1.407(0.664 \sim 2.984)$ & 0.513 & $1.377(0.526 \sim 3.606)$ & 0.162 & $0.403(0.109 \sim 1.498)$ \\
\hline \multicolumn{12}{|l|}{ DQB1 } \\
\hline *02 & $55(21.2)$ & $36(12.3)$ & $13(9.8)$ & $14(11.5)$ & $22(12.9)$ & $\underline{0.005}^{\mathrm{h}}$ & $1.908(1.206 \sim 3.018)$ & $\underline{0.005^{i}}$ & $2.456(1.288 \sim 4.682)$ & 0.707 & $0.872(0.427 \sim 1.782)$ \\
\hline$* 0301$ & $65(25.0)$ & $68(23.3)$ & $27(20.5)$ & $37(30.3)$ & $31(18.2)$ & 0.639 & $1.098(0.743 \sim 1.622)$ & 0.316 & $1.296(0.780 \sim 2.154)$ & $\underline{0.016 j}$ & $1.952(1.128 \sim 3.377)$ \\
\hline *0302 & $21(8.1)$ & $18(6.2)$ & $7(5.3)$ & $5(4.1)$ & $13(7.6)$ & 0.381 & $1.338(0.696 \sim 2.570)$ & 0.314 & $1.569(0.649 \sim 3.792)$ & 0.214 & $0.516(0.179 \sim 1.488)$ \\
\hline$* 0303$ & 31(11.9) & $44(15.1)$ & $23(17.4)$ & $18(14.8)$ & $26(15.3)$ & 0.282 & $0.763(0.466 \sim 1.250)$ & 0.135 & $0.642(0.357 \sim 1.152)$ & 0.899 & $0.959(0.500 \sim 1.839)$ \\
\hline$* 0306$ & $8(3.1)$ & $4(1.4)$ & $0(0.0)$ & $2(1.6)$ & $2(1.2)$ & 0.170 & $2.286(0.080 \sim 7.681)$ & 0.097 & $0.969(0.948 \sim 0.990)$ & 0.737 & $1.400(0.194 \sim 10.078)$ \\
\hline *04 & $21(8.1)$ & $19(6.5)$ & $9(6.8)$ & $12(9.8)$ & $7(4.1)$ & 0.478 & $1.262(0.663 \sim 2.405)$ & 0.658 & $1.201(0.534 \sim 2.701)$ & 0.051 & $2.540(0.970 \sim 6.654)$ \\
\hline$* 0501$ & $34(13.1)$ & $48(16.4)$ & $23(17.4)$ & $8(6.6)$ & $40(23.5)$ & 0.268 & $0.765(0.476 \sim 1.230)$ & 0.249 & $0.713(0.401 \sim 1.269)$ & $\underline{0.000}^{\mathrm{k}}$ & $0.228(0.103 \sim 0.507)$ \\
\hline$* 0601$ & $25(9.6)$ & $55(18.8)$ & $30(22.7)$ & $26(21.3)$ & $29(17.1)$ & $\underline{0.002^{1}}$ & $0.458(0.276 \sim 0.760)$ & $\underline{0.000}^{\mathrm{m}}$ & $0.362(0.203 \sim 0.646)$ & 0.359 & $1.317(0.730 \sim 2.374)$ \\
\hline
\end{tabular}

\section{Differences of HLA-DRB 1 and HLA-DQB 1 Polymorphisms between Group I and Group II}

In order to explore a putative link between specific HLA-DRB1 or HLA-DQB1 alleles and the early onset cervical SCCs, differences between younger cervical SCCs and older patients in allele distribution and frequency were further compared (Table 4). The most frequent allele detected for the DRB1 loci in group I was DRB1*09 $(20.5 \%, 25 / 122)$, whereas DRB1*08 (14.1\%, 24/170) and DRB1*12 $(14.1 \%, 24 / 170)$ were the two most frequent alleles found in group II. It is noteworthy that the allele frequencies of DRB $1^{*} 04$ and DRB1*09 were significantly higher in group I compared with those in group II, whereas DRB1*07 frequency in group I was significantly lower than that in group II. However, statistical significances were all disappeared after the application of Bonferroni's correction( $\left.\mathrm{Pc}_{\mathrm{C}} \mathrm{0.05}\right)$.

For the HLA-DQB1 loci, the allelic distribution found that DQB1*0301(30.3\%, 37/122) was the most frequent allele in group I, whereas DQB ${ }^{*} 0501(23.5 \%$, $40 / 170$ ) was the most frequent allele in group II. In addition to this, the frequency of DQB1*0501 was obviously lower in group I than that in group II, with a Pc value of 0.000 . A higher frequency of DQB1*0301 was found in group I than that in group II $(p=0.016)$, but no significant difference was kept after Bonferroni's correction $\left(\mathrm{Pc}_{\mathrm{C}}=0.128\right)$.

\section{The Relation of HLA-DRB 1 and HLA-DQB 1 Polymorphisms with HPV16 E6 Variants}

With the aim to identify a possible relationship of HLA-DRB1, DQB1 polymorphisms with specific E6 variants, we further compared the allele distribution in HPV16-positive cervical SCCs and healthy controls (Table 5).

Special associations between three alleles and certain E6 variants were observed. DRB1*09 and DQB1*0601 frequencies were remarkably increased among cervical SCCs with the A4 lineage compared with those among healthy controls, with a Pc value of 0.039 in the former and 0.000 in the latter. The allele frequency of DRB1*11 was obviously higher among cervical SCCs with the B variant compared with that among healthy controls, with a Pc value of 0.026 . Additionally, we found that DRB1 ${ }^{*} 08$ and DQB1 ${ }^{*} 0301$ frequencies were more common in cervical SCCs with the A1-3(350T) lineage compared with healthy controls $(p<0.05)$, but no significant difference was kept after Bonferroni's correction (Pc $>0.05)$. Similar tendencies were also observed in DQB1*0303 among cervical SCCs with the A4 lineage and in DRB1*12 in cervical SCCs with the B lineage compared with healthy controls $(p<0.05)$. But likewise, no significant difference was kept after Bonferroni's correction (Pc $>0.05)$. 
Table 5. The Relationship between HLA-DRB1, DQB1 Polymorphisms and HPV16 E6 Variants.

\begin{tabular}{|c|c|c|c|c|c|c|}
\hline \multirow[t]{2}{*}{ Alleles } & \multirow{2}{*}{$\begin{array}{l}\text { Healthy Controls } \\
2 n=260 \\
(\%)\end{array}$} & \multirow{2}{*}{$\begin{array}{l}\text { HPV16-positive Cervical } \\
\text { SCCs } \\
2 \mathrm{n}=132 \\
(\%)\end{array}$} & \multicolumn{4}{|l|}{ E6 Variants } \\
\hline & & & $\begin{array}{l}\text { A1-3 (350T) } \\
2 n=26 \\
(\%)\end{array}$ & $\begin{array}{l}\text { A1-3 (350G) } \\
2 \mathrm{n}=12 \\
(\%)\end{array}$ & $\begin{array}{l}\mathrm{A4} \\
2 \mathrm{n}=84 \\
(\%)\end{array}$ & $\begin{array}{l}\text { B } \\
2 n=10 \\
(\%)\end{array}$ \\
\hline \multicolumn{7}{|l|}{$\begin{array}{l}\text { DRB1 } \\
\end{array}$} \\
\hline *01 & $8(3.1)$ & $1(0.8)$ & $0(0.0)$ & $0(0.0)$ & $0(0.0)$ & $1(10.0)$ \\
\hline *03 & $23(8.8)$ & $9(6.8)$ & $4(15.4)$ & $0(0.0)$ & $5(6.0)$ & $0(0.0)$ \\
\hline *04 & $16(6.2)$ & $10(7.6)$ & $2(7.7)$ & $0(0.0)$ & $8(9.5)$ & $0(0.0)$ \\
\hline$* 07$ & $21(8.1)$ & $8(6.1)$ & $1(3.8)$ & $1(8.3)$ & $6(7.1)$ & $0(0.0)$ \\
\hline *08 & $29(11.2)$ & $23(17.4)$ & $\underline{7(27.0)^{\mathrm{a}}}$ & $4(33.3)$ & 11(13.1) & $1(10.0)$ \\
\hline$* 09$ & $26(10.0)$ & $19(14.4)$ & $\overrightarrow{0(0.0)}$ & $0(0.0)$ & $19(22.6)^{b}$ & $0(0.0)$ \\
\hline *10 & $8(3.1)$ & $2(1.5)$ & $0(0.0)$ & $0(0.0)$ & $2(2.4)$ & $0(0.0)$ \\
\hline *11 & $10(3.8)$ & $13(9.8)$ & $3(11.5)$ & $2(16.7)$ & $5(6.0)$ & $\underline{3(30.0)^{c}}$ \\
\hline *12 & $29(11.2)$ & 18(13.6) & $6(23.1)$ & $2(16.7)$ & $6(7.1)$ & $\underline{4(40.0)^{\mathrm{d}}}$ \\
\hline *13 & $18(6.9)$ & $0(0.0)$ & $0(0.0)$ & $0(0.0)$ & $0(0.0)$ & $0(0.0)$ \\
\hline *14 & $21(8.1)$ & $7(5.3)$ & $2(7.7)$ & $3(25.0)$ & $2(2.4)$ & $0(0.0)$ \\
\hline *15 & $35(13.5)$ & $16(12.1)$ & $0(0.0)$ & $0(0.0)$ & $16(19.0)$ & $0(0.0)$ \\
\hline *16 & $16(6.2)$ & $6(4.5)$ & $1(3.8)$ & $0(0.0)$ & $4(4.8)$ & $1(10.0)$ \\
\hline \multicolumn{7}{|l|}{ DQB1 } \\
\hline$* 02$ & $55(21.2)$ & $13(9.8)$ & $2(7.7)$ & $2(16.7)$ & $7(8.3)$ & $2(20.0)$ \\
\hline *0301 & $65(25.0)$ & $27(20.5)$ & $12(46.2)^{\mathrm{e}}$ & $5(41.6)$ & $7(8.3)$ & $3(30.0)$ \\
\hline *0302 & $21(8.1)$ & $7(5.3)$ & $0(0.0)$ & $0(0.0)$ & $7(8.3)$ & $0(0.0)$ \\
\hline *0303 & $31(11.9)$ & $23(17.4)$ & $3(11.5)$ & $1(8.3)$ & $18(21.4)^{\mathrm{f}}$ & $1(10.0)$ \\
\hline *0306 & $8(3.1)$ & $0(0.0)$ & $0(0.0)$ & $0(0.0)$ & $0(0.0)$ & $0(0.0)$ \\
\hline$* 04$ & $21(8.1)$ & $9(6.8)$ & $0(0.0)$ & $0(0.0)$ & $9(10.7)$ & $0(0.0)$ \\
\hline *0501 & $34(13.1)$ & $23(17.4)$ & $7(27.0)$ & $2(16.7)$ & $12(14.3)$ & $2(20.0)$ \\
\hline *0601 & $25(9.6)$ & $30(22.7)$ & $2(7.7)$ & $2(16.7)$ & $24(28.6) \mathrm{g}$ & $2(20.0)$ \\
\hline
\end{tabular}

Abbreviations: HPV, human papillomavirus; SCC, squamous cervical cancer

*The valid sample size was calculated as $2 \mathrm{n}$ for each case provided 2 separate alleles.

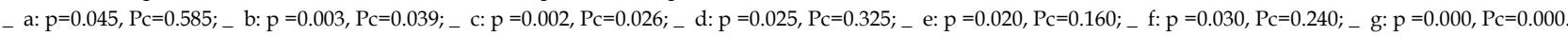

Table 6. The Distribution of DRB1, DQBI Alleles Associated with the A4 lineage between Group I and Group II.

\begin{tabular}{|c|c|c|c|c|c|}
\hline Alleles & Group I 2n=58(\%) & $\begin{array}{l}\text { Group II } \\
2 n=26(\%)\end{array}$ & $X^{2}$ & $p$ & OR(95\%CI) \\
\hline \multicolumn{6}{|l|}{ DRB1 } \\
\hline *03 & $0(0.0)$ & $5(19.2 \%)$ & 8.673 & $\underline{0.003}^{\mathrm{a}}$ & $1.238(1.026 \sim 1.494)$ \\
\hline *04 & $7(12.1 \%)$ & $1(3.8 \%)$ & 0.616 & 0.433 & $3.431(0.400 \sim 29.435)$ \\
\hline *07 & $3(5.2 \%)$ & $3(11.5 \%)$ & 0.347 & 0.556 & $0.418(0.079 \sim 2.227)$ \\
\hline *08 & $11(19.0 \%)$ & $0(0.0)$ & 4.130 & $\underline{0.042^{b}}$ & $0.810(0.715 \sim 0.918)$ \\
\hline *09 & $13(22.4 \%)$ & $6(23.1 \%)$ & 0.005 & 0.946 & $0.963(0.320 \sim 2.897)$ \\
\hline *10 & $2(3.4 \%)$ & $0(0.0)$ & - & - & - \\
\hline *11 & $2(3.4 \%)$ & $3(11.5 \%)$ & 0.903 & 0.342 & $0.274(0.043 \sim 1.748)$ \\
\hline *12 & $5(8.6 \%)$ & $1(3.8 \%)$ & 0.107 & 0.743 & $2.358(0.262 \sim 21.266)$ \\
\hline *14 & $2(3.4 \%)$ & $0(0.0)$ & - & - & - \\
\hline *15 & $9(15.5 \%)$ & $7(26.9 \%)$ & 0.865 & 0.352 & $0.499(0.163 \sim 1.529)$ \\
\hline *16 & $4(6.9 \%)$ & $0(0.0)$ & 0.669 & 0.413 & $0.931(0.868 \sim 0.999)$ \\
\hline \multicolumn{6}{|l|}{ DQB1 } \\
\hline *02 & $2(3.4 \%)$ & $5(19.2 \%)$ & 3.970 & $\underline{0.046} \underline{\underline{c}}^{\mathrm{c}}$ & $0.150(0.027 \sim 0.833)$ \\
\hline *0301 & $4(6.9 \%)$ & $3(11.5 \%)$ & 0.081 & 0.776 & $0.568(0.418 \sim 2.742)$ \\
\hline *0302 & $4(6.9 \%)$ & $3(11.5 \%)$ & 0.081 & 0.776 & $0.568(0.418 \sim 2.742)$ \\
\hline *0303 & $11(19.0 \%)$ & $7(26.9 \%)$ & 0.675 & 0.411 & $0.635(0.214 \sim 1.884)$ \\
\hline *04 & $9(15.5 \%)$ & $0(0.0)$ & 3.042 & 0.081 & - \\
\hline *0501 & $8(13.8 \%)$ & $4(15.4 \%)$ & 0.000 & 1.000 & $0.880(0.240 \sim 3.231)$ \\
\hline *0601 & $20(34.5 \%)$ & $4(15.4 \%)$ & 3.208 & 0.073 & $2.895(0.876 \sim 9.563)$ \\
\hline
\end{tabular}

\section{The Distribution of HLA-DRB 1 and HLA-DQB 1 Alleles Associated with the HPV16 A4 Lineage between Young Cervical SCCs and Older Patients}

As the A4 lineage was the most prevalent type observed among patients with HPV16-positive cervical SCC in both groups, and was the exclusive genotype detected in group I, we further compared the distribution of DRB1, DQB1 alleles associated with the A4 lineage among cervical SCC patients (Table 6).

A significant association was observed for DRB1*03, with a Pc value of 0.039 . This allele was not found among cervical SCCs positive for the A4 lineage in group I, whereas it was detected among those in group II with the percentage of $19.2 \%(5 / 26)$. 
It is worthwhile to note that DRB1*08 frequency was significantly increased among cervical SCCs positive for the A4 lineage in group I compared with those in group II $(p<0.05)$. By contrast, DQB1*02 frequency was decreased among patients with the A4 lineage in group I $(p<0.05)$. However, statistical significances were lost after adjustment made for the other alleles $\left(\mathrm{Pc}_{\mathrm{C}}>0.05\right)$. Additionally, allele frequencies of DQB1*04 and DQB1*0601were increased among cervical SCCs with the A4 lineage in group I, but no statistical significance was observed $(p>0.05)$. Although HLADRB1*09 frequency was significantly higher in cervical SCCs with A4 lineage than controls, no significant difference was found between group I and group II $(p=0.946)$.

\section{Discussion}

It is generally acknowledged that the time course from high-risk HPV infection to the development of invasive cervical cancer usually takes about 15 20 years [25]. The obvious earlier onset age of cervical SCC among young patients suggests the existence of some other important factors associated with the accelerated carcinogenesis. It is possible that genetic factors influence viral persistence, and specific more virulent variants of HPV may play important roles in early onset cervical SCCs. In the present study, we analyzed the distribution of HPV16 E6 sequence variants and HLA-DRB1, -DQB1 allele frequencies among a cervical SCC population in China. The dissimilarities of DRB1, DQB1 polymorphisms between young cervical SCCs and older cases and the correlation with HPV16 E6 variants were also analyzed.

In agreement with our previous study, the A4 lineage was also the most prevalent genotype observed (63.6\%). We observed that the E6 variants distributed significantly different between young cervical SCC patients and older cases. The A4 lineage was the exclusive type observed in young cervical SCCs, and its prevalence was significantly higher than that of older cases $(82.9 \%$ vs. $41.9 \%, p<0.05)$. The diverse distribution of the A4 lineage between younger cervical SCCs and older ones occurred in the same region indicated that this lineage was probably more carcinogenic in young women. Results of a few studies $[19,26]$ also indicated that young women appeared to be more vulnerable or susceptible to certain E6 variant genotypes in a specific population. The host genetic diversity, for example the HLA alleles polymorphisms may be used to explain this difference. Some HLA molecules might be more efficient in presenting epitopes from certain E6 variant genotypes and thus determine its potential carcinogenicity [23].
Previous studies from diverse ethnic populations have analyzed the possible correlation of HLA II molecules with cervical cancer $[22,27,28]$. Although the same HLA class II alleles may be detected frequently to be associated with the occurrence of cervical cancer in different populations, there's still no consensus on which HLA II alleles may increase the risk of cervical carcinoma in any population. In the present study, allele frequencies of DRB1*11 and DQB1*0601 increased significantly in cervical SCCs when compared with healthy controls, whereas DRB1*13 and DQB1*02 were significant decreased in cervical SCCs. In addition, a similar association was observed for DRB1*13 and DQB1*0601 in HPV16positive cervical SCCs when compared with healthy controls. These results suggested the protective effect of DRB1*13 and the predisposing effect of DQB1 ${ }^{*} 0601$ may be specific for HPV16 in this study population.

To further explore possible accelerating factors associated with early onset cervical SCC in young population, the investigation of both HPV intra-typic variations and HLA-DRB1, HLA-DQB1 polymorphisms between young cervical SCCs and older patients, as demonstrated in this study, is necessary. To the best of our current knowledge, this study is the first comprehensive investigation regarding the difference of DRB1, DQB1 polymorphisms between young cervical SCCs and older patients. The results indicated different distribution of certain DRB1, DQB1 alleles between young cervical SCCs and older ones. The allele frequencies for DRB1*04, DRB1*09 and DQB1*0301 among young cervical SCCs were significantly increased when compared with older ones $(p<0.05)$. On the other hand, DRB1*07 and DQB1*0501 in young cervical SCCs were significantly decreased when compared with older ones $(p<0.05)$. After adjustment for the other alleles was made, statistical significance was kept only for DQB1* 0501(Pc<0.05), thereby indicating its protective effect. This result suggests that young women who are carriers of HLA-DQB1*0501 allele may have a relatively lower risk for the development of an early onset cervical SCC.

Based on the above findings between young cervical SCCs and older patients, we hypothesize that certain DRB1, DQB1 alleles may be associated to special HPV16 E6 variants, and this putative relationship may contribute to the obviously accelerated carcinogenesis in this young Chinese population with cervical SCC.

Concerning the possible correlation between the E6 variant genotype and HLA polymorphisms in the development of cervical cancer, a number of studies from different ethnic populations have found that certain HLA class II alleles related with specific HPV 
variants showed a predisposing effect or a protective effect in cervical carcinogenesis $[23,24,29,30]$. However, we know little about a possible relation between specific HPV16 E6 variant and HLA-DRB1, HLADQB1 alleles in the obviously accelerated carcinogenesis among young women with cervical SCC.

In the present study, we found that the allele frequencies of DRB1*09 and DQB1*0601 were significantly higher among cervical SCC patients with the A4 lineage when compared with healthy controls. This result suggested that carriers of DRB1 ${ }^{*} 09$ or DQB1*0601 alleles may be at increased risk for developing cervical SCC once infected with the A4 lineage. Additionally, DRB1*11 was found to be positively associated with the $B$ variant.

As we have noticed before, the A4 lineage was not only the most common type of E6 variant observed among this study population, but it was also the unique variant found in young cervical SCCs. Then we further extended the type-specific analysis concerning the distribution of DRB1, DQB1 alleles associated with the A4 lineage between young cervical SCCs and older patients. The DRB1*03 was not detected among young patients positive for the A4 lineage. A significant higher frequency of DRB1*03 was found among older patients positive for the A4 lineage compared with that among young patients $(\operatorname{Pc}<0.05)$. This result indicates that young women who are carriers of HLA-DRB1*03 allele may at lower risk of developing an early onset cervical SCC associated with the HPV16 A4 lineage in this Chinese population. In addition to this, an increased frequency of DRB1*08 and a decreased frequency of DQB1*02 were also observed among young patients positive for the A4 lineage compared with those among older patients, with $p$ values of $<0.05$. However, statistical significance was not kept upon Bonferroni's correction. These results suggested that these alleles may display a possible predisposing or protective effect in the carcinogenesis of an early onset cervical SCC associated with the HPV16 A4 lineage in this Chinese population. Failure to get a significant correlation may due to the modest sample size.

\section{Conclusion}

The present data not only provided evidence for the difference of DRB1, DQB1 polymorphisms between young patients with cervical SCC and older ones, but also gave clues on the possible relationship between certain HPV16 E6 variants and HLA class II alleles. In addition, our data further implied that certain HLA-DRB1 and HLA-DQB1 alleles may be related to the A4 lineage among young cervical SCCs, which was the unique HPV16 E6 variant found in young patients. These results suggested that the early onset cervical cancer in this study population was, at least in part, affected by specific association between certain HPV variant and genetic polymorphisms of HLA. In order to further verify the possible link between certain HPV16 E6 variant and HLA-DRB1, HLA-DQB1 alleles and the relationship with the early occurred cervical SCCs in young population, studies on larger sample size will be required. Such information may help to elucidate the pathogenic factors that associated with young women cervical SCCs, and thus to provide more accurate approaches for better prophylaxis and treatment.

\section{Abbreviations}

HPV: human papillomavirus; HLA: human leukocyte antigen; ICC: invasive cervical cancer; SCC: squamous cell carcinoma; SSP: sequence specific primers.

\section{Acknowledgment}

This work was supported by grants from the Natural Science Foundation of Zhejiang Province, China (Grant No. Y204080).

\section{Competing Interests}

The authors have declared that no competing interests exist.

\section{References}

[1] Ferlay J, Soerjomataram I, Dikshit R, et al. Cancer incidence and mortality worldwide: Sources, methods and major patterns in GLOBOCAN 2012. Int J Cancer. 2015; 136: E359-E386

[2] de Sanjose S, Serrano B, Castellsague X, et al. Human papillomavirus (HPV) and related cancers in the Global Alliance for Vaccines and Immunization (GAVI) countries. A WHO/ICO HPV Information Centre Report. Vaccine. 2012; 30(Suppl 4): D1-83

[3] Foley G, Alston R, Geraci M, et al. Increasing rates of cervical cancer in young women in England: an analysis of national data 1982-2006. Br J Cancer. 2011; 105: 177-184

[4] Li S, Hu T, Lv W, et al. Changes in Prevalence and Clinical Characteristics of Cervical Cancer in the People's Republic of China: A Study of 10,012 Cases From a Nationwide Working Group. Oncologist. 2013; 18: 1101-1107

[5] Adegoke O, Kulasingam S, Virnig B. Cervical cancer trends in the United States: a 35-year population-based analysis. J Womens Health (Larchmt). 2012; 21: 1031-1037

[6] Kokawa K, Takekida S, Kamiura S, et al. The incidence, treatment and prognosis of cervical carcinoma in young women: a retrospective analysis of 4,975 cases in Japan. Eur J Gynaecol Oncol. 2010; 31: 37-43

[7] Miyagi E, Motoki Y, Asai-Sato M, et al. Web-based Recruiting for a Survey on Knowledge and Awareness of Cervical Cancer Prevention Among Young Women Living in Kanagawa Prefecture, Japan. Int J Gynecol Cancer. 2014; 24: 1347-1355

[8] de Freitas AC, Coimbra EC, Leitao Mda C. Molecular targets of HPV oncoproteins: potential biomarkers for cervical carcinogenesis. Biochim Biophys Acta. 2014; 1845: 91-103

[9] Azuma Y, Kusumoto-Matsuo R, Takeuchi F, et al. Human Papillomavirus Genotype Distribution in Cervical Intraepithelial Neoplasia Grade 2/3 and Invasive Cervical Cancer in Japanese Women. Jpn J Clin Oncol. 2014; 44: 910-917

[10] de Sanjose S, Quint WG, Alemany L, et al. Human papillomavirus genotype attribution in invasive cervical cancer: a retrospective cross-sectional worldwide study. Lancet Oncol. 2010; 11: 1048-1056

[11] Tamegao-Lopes BP, Sousa-Junior EC, Passetti F, et al. Prevalence of human papillomavirus infection and phylogenetic analysis of HPV-16 E6 variants among infected women from Northern Brazil. Infect Agent Cancer. 2014; 9: 1-7

[12] Boumba LM, Assoumou SZ, Hilali L, et al. Genetic variability in E6 and E7 oncogenes of human papillomavirus Type 16 from Congolese cervical cancer isolates. Infect Agent Cancer. 2015; 10: 15 
[13] Ortiz-Ortiz J, Alarcon-Romero Ldel C, Jimenez-Lopez MA, et al. Association of human papillomavirus 16 E6 variants with cervical carcinoma and precursor lesions in women from Southern Mexico. Virol J. 2015; 12: 1-14

[14] Burk RD, Harari A, Chen Z. Human papillomavirus genome variants. Virology. 2013; 445: 232-243

[15] Lee CW, Bae JH, Lee SJ, et al. Distribution of human papillomavirus type 16 E6 and E7 gene variants in the progression of cervical dysplasia in Korean women. J Obstet Gynaecol Res. 2011; 37: 1320-1326

[16] Ding T, Wang X, Ye F, et al. Distribution of human papillomavirus 16 E6/E7 variants in cervical cancer and intraepithelial neoplasia in Chinese women. Int J Gynecol Cancer. 2010; 20: 1391-1398

[17] Matsumoto K, Yoshikawa H. Human papillomavirus infection and the risk of cervical cancer in Japan. J Obstet Gynaecol Res. 2013; 39: 7-17

[18] Sun Z, Lu Z, Liu J, et al. Genetic variations of E6 and long control region of human papillomavirus type 16 from patients with cervical lesion in Liaoning, China. BMC Cancer. 2013; 13: 459

[19] Hu Y, Zhu YY, Zhang SH, et al. Human Papillomavirus Type 16 E6 Gene Variations in Young Chinese Women With Cervical Squamous Cell Carcinoma. Reprod Sci. 2010; 18: 406-412

[20] Chen D, Gyllensten U. A cis-eQTL of HLA-DRB1 and a frameshift mutation of MICA contribute to the pattern of association of HLA alleles with cervical cancer. Cancer Med. 2014; 3(2): 445-452

[21] Ben Othmane Y, Ghazouani E, Mezlini A, et al. HLA class II susceptibility to cervical cancer among Tunisian women. Bull Cancer. 2012; 99(9): 81-86

[22] $\mathrm{Hu} \mathrm{JM}$, Sun Q, Li L, et al. Human leukocyte antigen-DRB1*1501 and DQB1*0602 alleles are cervical cancer protective factors among Uighur and Han people in Xinjiang, China. Int J Clin Exp Pathol. 2014; 7: 6165-6171

[23] de Araujo Souza PS, Maciag PC, Ribeiro KB, et al. Interaction between polymorphisms of the Human Leukocyte Antigen and HPV-16 Variants on the risk of invasive cervical cancer. BMC Cancer. 2008; 8: 1-9

[24] Wu Y, Liu B, Lin W, et al. HPV16 E6 variants and HLA class II polymorphism among Chinese women with cervical cancer. J Med Virol .2007; 79: 439-446

[25] Sasagawa T, Takagi H, Makinoda S. Immune responses against human papillomavirus (HPV) infection and evasion of host defense in cervical cancer. J Infect Chemother. 2012; 18: 807-815

[26] Qmichou Z, Khyatti M, Berraho M, et al. Analysis of mutations in the E6 oncogene of human papillomavirus 16 in cervical cancer isolates from Moroccan women. BMC Infect Dis. 2013; 13: 378

[27] Wei LZ, Wang HL, Liu X, et al. Meta-Analysis on the Relationship between HLA-DRBl Gene Polymorphism and Cervical Cancer in Chinese Population. PLoS ONE. 2014; 9: e88439.

[28] Ben Othmane Y, Ghazouani E, Mezlini A, et al. HLA class II susceptibility to cervical cancer among Tunisian women. Bull Cancer. 2012; 99:81-86.

[29] Matsumoto K, Yasugi T, Nakagawa S, et al. Human papillomavirus type 16 E6 variants and HLA class II alleles among Japanese women with cervical cancer. Int J Cancer. 2003; 106: 919-922.

[30] de Araujo Souza PS, Sichero L, Maciag PC. HPV variants and HLA polymorphisms: the role of variability on the risk of cervical cancer. Future Oncol. 2009; 5: 359-370. 